\title{
Use of Spectral Kurtosis for improving signal to noise ratio of acoustic emission signal from defective bearings
}

\author{
C. Ruiz-Cárcel ${ }^{1}$, E. Hernani-Ros ${ }^{1}$, Y. Cao ${ }^{1}, \mathrm{D} . \mathrm{Mba}^{1}$ \\ (1)School of Engineering, Cranfield University, Building 52 School of Engineering, MK430AL (UK) \\ Cristóbal Ruiz Carcel \\ Email: c.ruizcarcel@cranfield.ac.uk \\ Phone number: +44 (0) 1234754639
}

\begin{abstract}
The use of Acoustic Emission (AE) to monitor the condition of roller bearings in rotating machinery is growing in popularity. This investigation is centred on the application of Spectral Kurtosis (SK) as a denoising tool able to enhance the bearing fault features from an AE signal. This methodology was applied to AE signals acquired from an experimental investigation where different size defects were seeded on a roller bearing. The results suggest that the signal to noise ratio can be significantly improved using SK.
\end{abstract}

\section{Keywords}

Acoustic emission, signal-to-noise ratio, spectral Kurtosis, roller bearings.

\section{Introduction}

\section{Vibration-based condition monitoring of bearings}

Rolling element bearings are common components in rotating machinery, and monitoring their condition can be vital to ensure the integrity and smooth operation of the machine. Over the last decades, vibration monitoring has been the most widely used method to detect and diagnose bearing faults, and several techniques have been developed to enhance the fault signature in the time or frequency domain. One example of these techniques is the use of adaptive and self-adaptive filters to remove background noise from the signals obtained through computer simulations and experimental data [1-4]. Another advanced techniques usually applied to augment the detectability of bearing faults are Fisher Linear Discriminant (FLD), K-Nearest Neighbor (KNN) and Support Vector Machine (SVM) [5]. Other investigators have developed techniques such as Envelope Order Tracking to allow the detection of faults in bearings working under varying speed conditions [6].

One of the most effective ways to detect faults in bearings from vibrational data is to monitor the value of the Kurtosis of the acquired signal. Kurtosis is defined mathematically as the fourth moment of a probability density function (see eq. 1), and it gives a measurement of the degree of impulsiveness in the signal, which is normally related with bearing faults [7].

$$
K=\frac{\int_{-\infty}^{\infty}[x-\mu]^{4} p(x) d x}{\sigma^{4}}
$$

In real applications, other sources of vibration or background noise often mask the bearing fault features in the signal and as a result the Kurtosis may be unable to capture the peakiness of the faulty signal. In such cases, the Kurtosis as a global indicator is not useful however

Published by Springer-Verlag. This is the Author Accepted Manuscript.

1 This article may be used for personal use only. The final published version (version of record) is available online at 10.1007/s11668-014-9805-7. Please refer to any applicable publisher terms of use. 
better results may be obtained if the Kurtosis value was calculated across different frequency bands [8]. The Kurtogram, developed by Antoni and Randall [8] is a representation of the Kurtosis value as a function of the frequency and the bandwidth of the different frequency bands where it is calculated. Using the Kurtogram it is possible to identify the frequency band where the Kurtosis is maximum. This information can be used to design a filter which extracts the part of the signal with the highest level of impulsiveness, enhancing the bearing fault signal from the raw signal. This technique has been already applied successfully by different researchers in bearing fault detection and diagnosis [9-11].

\section{Condition monitoring of bearings based on Acoustic Emission}

Despite of the success of vibration-based methodologies, over the last decades bearing condition monitoring techniques based on Acoustic Emission (AE) have become very popular [12-31]. AE has demonstrated to be a very powerful tool for fault detection and diagnosis particularly in bearings, and some recent studies $[13 ; 32 ; 33]$ reported that AE can be more sensitive in detecting incipient faults in bearings over other methodologies based on vibration data. Matthews J.R. [34] defined AE as transient elastic waves generated from a rapid release of energy, caused by a deformation or damage within or on the surface of a material. In the particular case of bearings, AE is generated by the interaction of two surfaces that are in relative movement to each other. The interaction of the rolling elements with a defective surface will generate an AE burst superimposed onto continuous AE background noise, as seen in Figure 1.

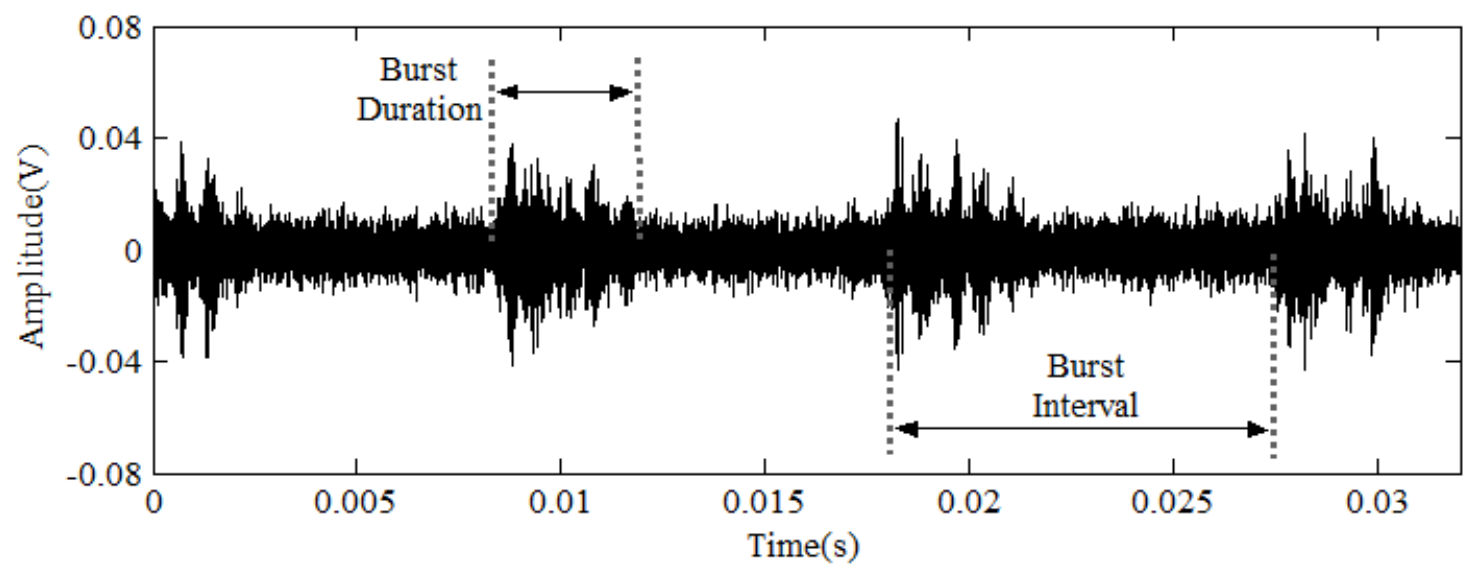

Figure 1.

Typical AE bursts associated to an outer race defect

Theoretically, it is possible to identify the defect source just by measuring the interval of time between each burst. This interval is related with a particular defect frequency that can be easily calculated from the bearing geometry, indicating the origin of the bursts. This technique has been successfully applied by different researchers $[12 ; 33]$ to identify outer race defects, but there have been reported difficulties in identifying inner race defects using AE $[12 ; 20 ; 35]$.

In addition, Al-Dossary, $\mathrm{S}$. et al. [12] reported that there is a strong correlation between the AE burst duration (see Figure 1) and the actual size of the defect; undertaken in an investigation where defects of different size were seeded on a roller bearing. In that experiment, the measured burst duration was close to the theoretical time duration of the roller passing over the defect, irrespective of the speed and load condition. This fact 
demonstrated that AE can be used not only to detect and diagnose bearing faults, but also to obtain information about the defect severity. The main problem faced by the authors was the relatively low signal to noise ratio found in the signal, especially in those tests where the defect size was small. This issue made it difficult to identify the instant when an AE burst overcame background noise level; essentially the point where the burst goes over background noise level indicating its start or end.

Eftekharnejad et al. [33] was the first to report that it is also possible to effectively increase the signal to noise ratio and enhance the bearing fault features in an AE signal using Spectral Kurtosis (SK) and the Kurtogram. The main aim of this paper is to complement the work of Al-Dossary et al. [12] by post-processing the AE signal acquired during the tests in order to increase the signal to noise ratio, reinforcing the novel idea of applying SK in AE signals as Eftekharnejad et al. [33] postulated recently.

\section{Experimental methodology}

\section{Test rig set-up}

The data used for this investigation is the same used by Al-Dossary et al. in [12]. In the test-rig, the test bearing is mounted on a shaft driven by an electric motor. The shaft is supported by two large slave bearings and the load is applied to the test bearing using a hydraulic cylinder (see Figure 2).

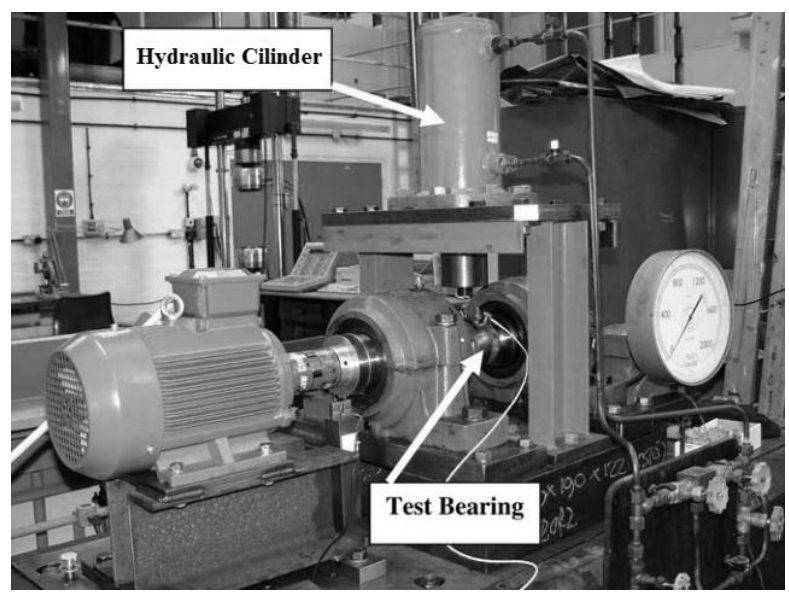

Table 1:

Main bearing dimensions

\begin{tabular}{ll}
\hline No. of rolling elements & 10 \\
Roller Diameter & $12 \mathrm{~mm}$ \\
External Diameter & $84 \mathrm{~mm}$ \\
Pitch Diameter & $68 \mathrm{~mm}$ \\
\hline
\end{tabular}

Figure 2.

Layout of experimental test-rig (11)

The test bearing chosen for the experiment was a Cooper cylindrical roller type 01B40MEX, and its main dimensions are summarized in Table 1. The selected sensor was a piezoelectric "Physical Acoustic Corporation type WD" with an operating frequency rate of $100-100 \mathrm{kHz}$, which was mounted on the top half of the bearing housing. The acquired signal was sampled at $8 \mathrm{MHz}$ for a rotating speed of $1500 \mathrm{rpm}$, and then amplified at $40 \mathrm{~dB}$. Nearly 250,000 points where acquired for each measurement, which corresponds approximately to a signal of $30 \mathrm{~ms}$.

In the original experiment [12], 4 different bearings were tested under different load and speed conditions. Defects of different sizes were seeded in such a manner as to understand how the defect size influences the AE waveform. For that purpose, different bearings with seeded faults were tested at 300,1500 and $3000 \mathrm{rpm}$ under loads of $2.7 \mathrm{kN}, 5.3 \mathrm{kN}$ and $8 \mathrm{kN}$. 
For this paper, only the AE signal acquired from one of those test bearings is analysed, where the load applied to the bearing was $5.3 \mathrm{kN}$ and the rotational speed of the motor was 1500 $\mathrm{rpm}$. The defects on the outer race were made using an electric engraver with a carbide tip. A total of 9 different defect sizes were employed: see Table 2 where L represents the length measured in the circumferential direction and $\mathrm{W}$ is the width across the bearing race. After each test, the bearing was disassembled and a next seeded bearing assembled. The rig was run for 15 minutes prior to the data acquisition in order to bring it up to thermal equilibrium.

Table 2:

\begin{tabular}{ll}
\multicolumn{2}{c}{ Incremental defect sizes (outer race) } \\
Defect & Size $(\mathbf{L} \times \mathbf{W}) \mathbf{~ m m}$ \\
\hline D1 & Circle $\mathrm{D}=0.5 \mathrm{~mm}$ \\
D2 & $0.9 \times 2.5$ \\
D3 & $0.9 \times 4$ \\
D4 & $0.9 \times 8$ \\
D5 & $0.9 \times 12$ \\
D6 & $3 \times 12$ \\
D7 & $5 \times 12$ \\
D8 & $7 \times 12$ \\
D9 & $9 \times 12$ \\
\hline
\end{tabular}

\section{Methodology}

As mentioned in the introduction section, the aim of this paper is to improve the signal to noise ratio of $\mathrm{AE}$ signals in order to enhance the bearing fault features in the acquired signal. The main benefit of using SK is that the frequency band where the signal is band-pass filtered to enhance the bearing defect signature is selected automatically depending on the original signal characteristics. The Kurtogram is used in the first instance to locate the frequency region where the Kurtosis is maximum, indicating a higher level of impulsiveness. For this investigation, the algorithm proposed by Antoni, J. [36; 37]for the fast computation of the Kurtogram was used, and the Kurtograms obtained for the different tests performed are shown in Figure 6.

The information provided by the Kurtogram is used to design a band-pass filter for the purpose of enhancing the impulsiveness of the original signal. In this work FIR filters were employed to filter the signal, and the filters were designed using Matlab Filter Design and Analysis Tool. The characteristics of the filters (according to Figure 3) were: $A_{\text {pass }}=1 \mathrm{~dB}$, $A_{\text {stop } 1}=60 \mathrm{~dB}, A_{\text {stop } 2}=80 \mathrm{~dB}$, and finally $F_{\text {stop } 1}$ and $F_{\text {stop2 }}$ were always selected $2 \mathrm{kHz}$ below and above the frequency band limits

The information provided by the Kurtogram for the different signals analysed (central frequency $\mathrm{F}_{\mathrm{c}}$, frequency resolution $\Delta f$ and maximum Kurtosis (Kmax) is summarized in

Table 3. Kmax represents the maximum Kurtosis value found in all the frequency regions inspected for all the levels analysed. 


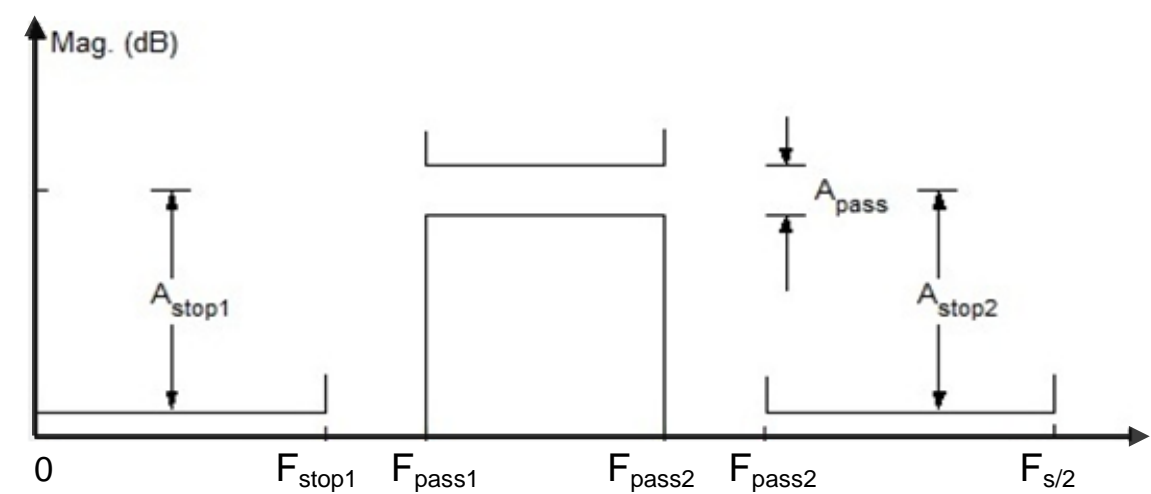

Figure 3.

Filter description

Table 3:

Information extracted from Kurtogram and filter design toolbox

\begin{tabular}{ccccc} 
Defect & $\mathbf{F c}(\mathbf{H z})$ & $\Delta \mathbf{f}(\mathbf{H z})$ & $\mathbf{K} \mathbf{m a x}$ & $\begin{array}{l}\text { Frequency } \\
\text { Band(Hz) }\end{array}$ \\
\hline D1 & $1,000,000$ & $2,000,000$ & 1.2 & $0-2,000,000$ \\
D2 & 531,250 & $20,833.33$ & 5.4 & $521,000-542,000$ \\
D3 & 46,875 & 31,250 & 16.9 & $31,250-62,500$ \\
D4 & $52,083.33$ & $20,833.33$ & 34.2 & $41,660-62,500$ \\
D5 & $70,312.5$ & 15,625 & 18.2 & $62,500-78,120$ \\
D6 & 78,125 & 31,250 & 17.7 & $62,500-93,750$ \\
D7 & 31,250 & 62,500 & 45 & $0-62,500$ \\
D8 & $54,687.5$ & 15,625 & 5 & $46,880-62,500$ \\
D9 & $72,916.67$ & $20,833.33$ & 6.8 & $62,500-8,330$ \\
\hline
\end{tabular}

In addition, the effectiveness of the denoising effect of SK on AE signals has been quantified by comparing the signal to noise ratio (SNR) of the original and filtered signals. The signal to noise ratio has been estimated using the Crest Factor (CF), which is defined as the ratio between the maximum absolute value and the signal r.m.s., giving an indication of the peak-to-average ratio [33]. The CF has already been successfully used as an indicator of damage severity in other investigations about $\mathrm{AE}$ applied to bearing fault detection $[12 ; 18$; $33 ; 38]$.

\section{Results and discussions}

\section{Time domain}

Figure 4 shows the original signals acquired for the 9 defects tested in the left column and the corresponding filtered signals in the right column (the $\mathrm{Y}$ axis represents the amplitude in Volts). Each waveform represents $30 \mathrm{~ms}$ of acquired signal, which corresponds approximately $3 / 4$ of one revolution. The outer race defect frequency is 4.1 times the shaft speed; therefore 3 or 4 burst spaced at $9.75 \mathrm{~ms}$ are expected to be visible in captured time 
waveform. The Kurtograms for the different signals are attached in Figure 6., and details about the information provided by SK can be found in

Table 3.

Original

D1

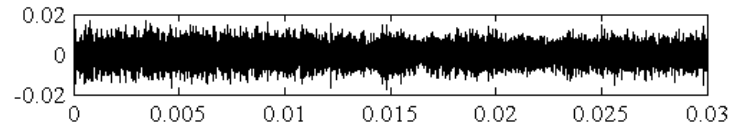

D2

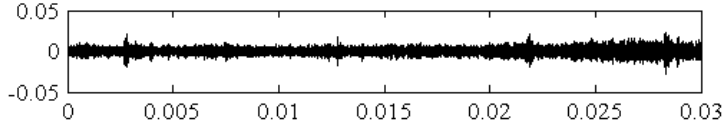

D3

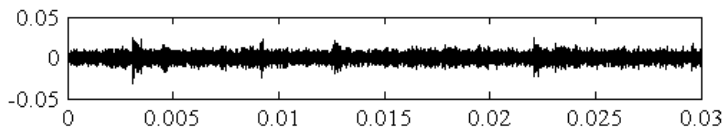

D4

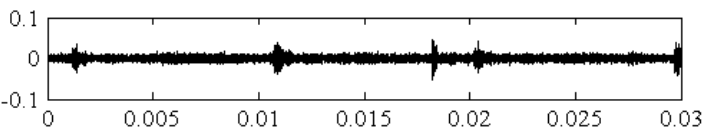

D5

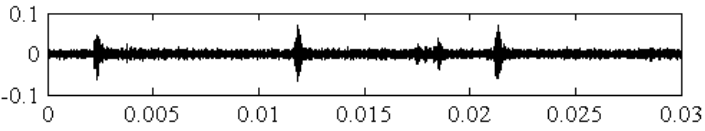

D6

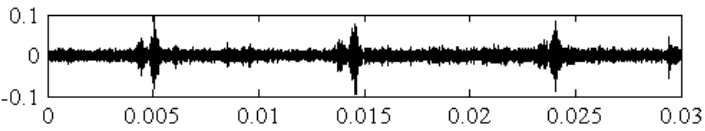

D7

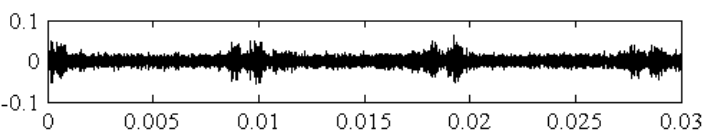

D8

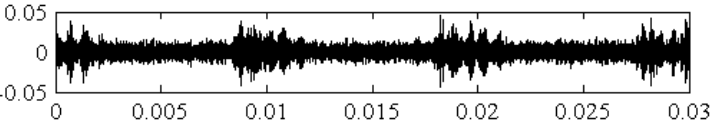

D9

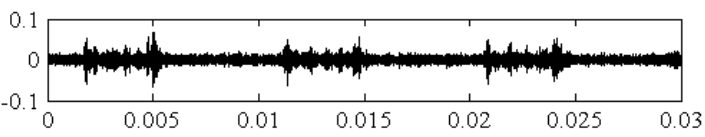

Time (s)
Filtered
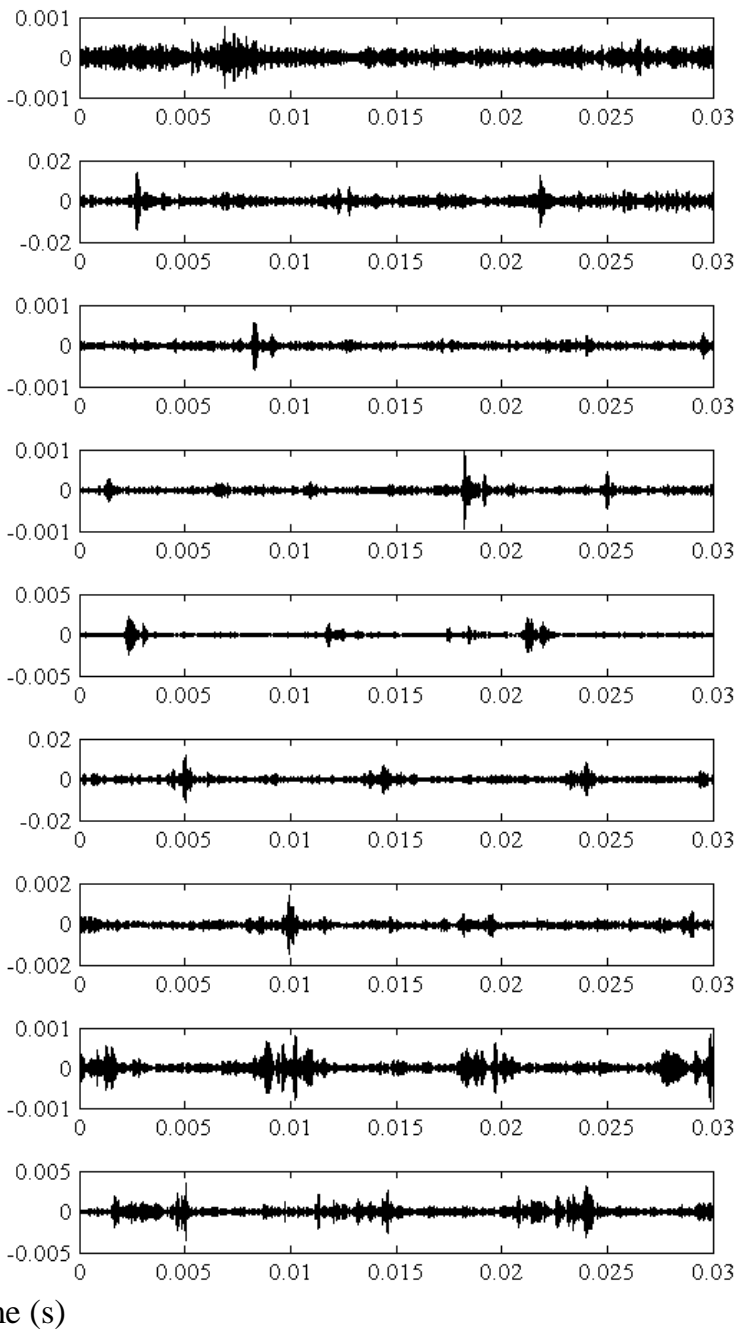

Figure 4.

Signal amplitude (Volts) of the original (left) and filtered signals (right)

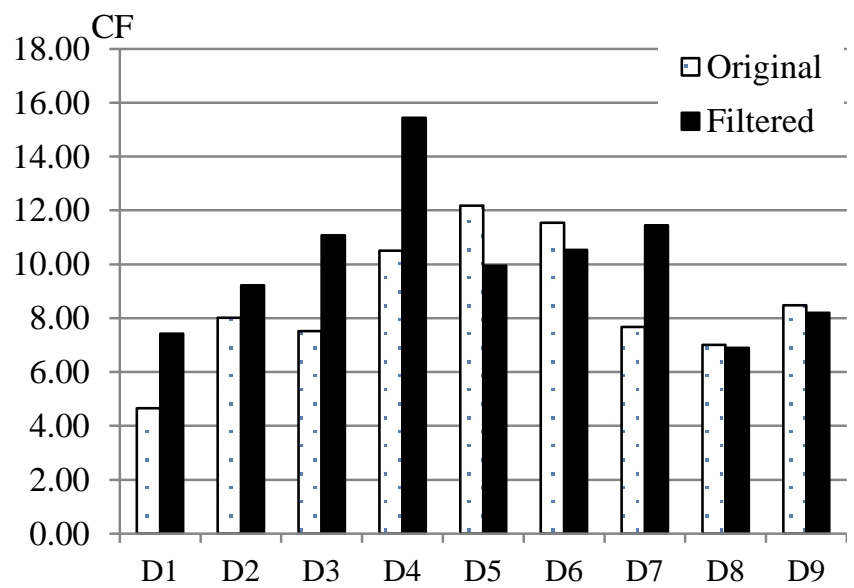

Table 4:

$\mathrm{CF}$ for the original and filtered signals

Original Filtered Improvement \%

$\begin{array}{cccc}\text { D1 } & 4.65 & 7.43 & 59.71 \\ \text { D2 } & 8.01 & 9.22 & 15.07 \\ \text { D3 } & 7.53 & 11.07 & 47.13 \\ \text { D4 } & 10.50 & 15.45 & 47.07 \\ \text { D5 } & 12.18 & 9.95 & -18.29 \\ \text { D6 } & 11.54 & 10.54 & -8.66 \\ \text { D7 } & 7.67 & 11.46 & 49.30 \\ \text { D8 } & 7.01 & 6.90 & -1.66\end{array}$

Figure 5.

Crest factor for the original and filtered signals 

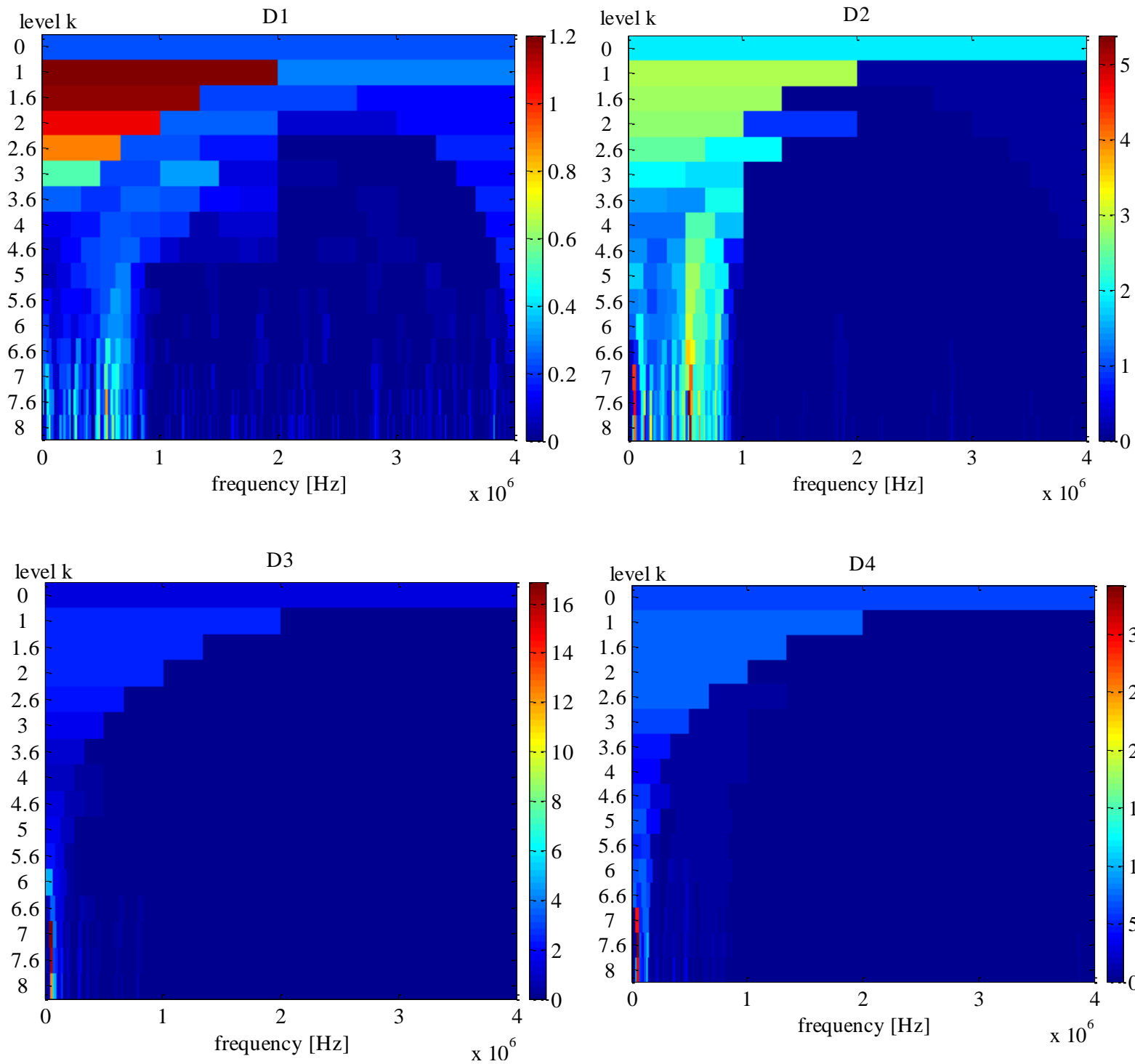

level k

D4

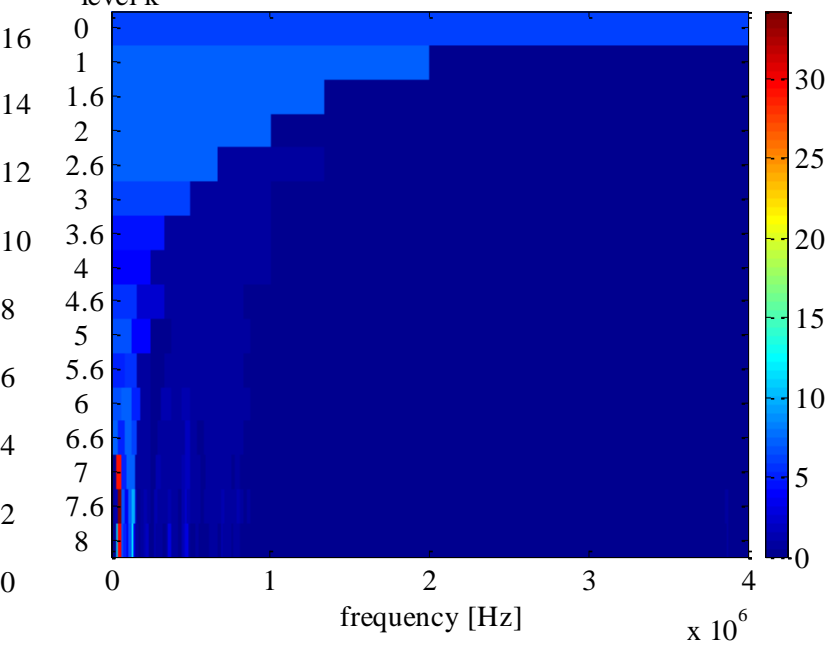



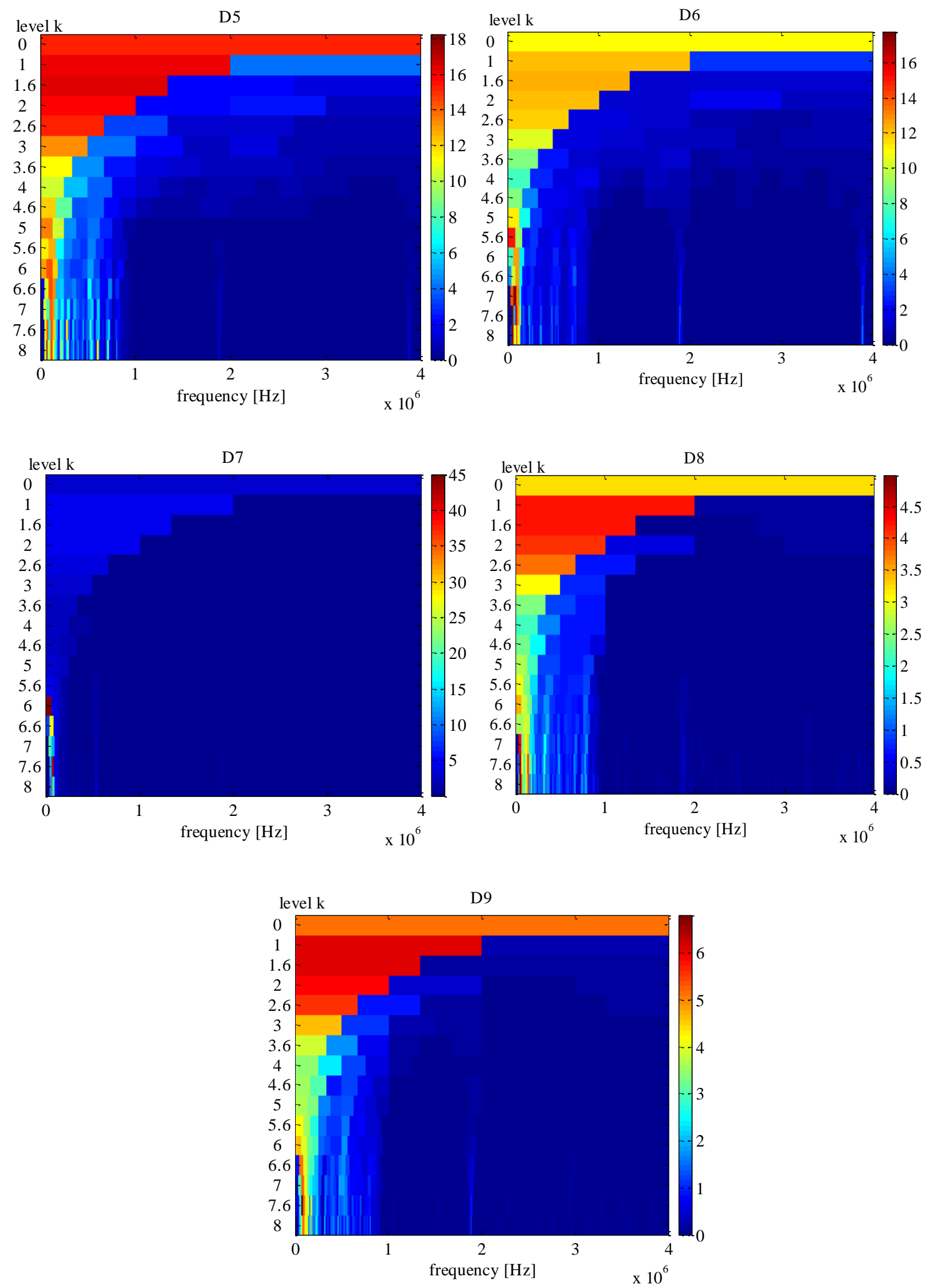

Figure 6.

Kurtograms 
Intuitively, it is possible to see in Figure 4 that level of background noise in the filtered signals has been reduced when compared with the original signals. The same bursts are visible in the filtered signal though with lower amplitude due to the different voltage ranges. In the filtered signals the signal to noise ratio is higher, especially in the smaller defects tested, showing the denoising capability of SK. This fact can be observed for example in D2 (Figure 4), where the bursts happening approximately $0.0025,0.0125$ and 0.0225 seconds after the beginning of the measurement are much more visible over the background noise in the filtered signal than in the original signal. Specifically the SNR in this observation has improved by $15 \%$. The same effect can be observed in tests D3 to D9, where the peaks in the signal generated by the rolling element passing over the defects are more visible over the background noise compared with the original signals. This improvement can facilitate the task of identifying those peaks during the early stages of degradation when the peak intensity is lower, or in applications where the background noise is significantly high. In the smallest defect tested (D1) there are no visible bursts in the original signal and SK was not able to extract the impulsive part of the signal. Consequently it is not possible to identify any bursts in the filtered or the original signal for the first defect.

Figure 5 shows the crest factor in the original and filtered signals for each defect tested. The same information is detailed in Table 4, including the percentage of improvement achieved in the filtered signal. The improvement in the signal to noise ratio is relatively high in the smaller defects tested (D1 to D4) and in D7, but it is insignificant in the rest of defects and much worse in the case of D5. The average percentage of improvement in the CF excluding D1 is $15.8 \%$. A possible explanation for this phenomenon is the change in the probability density function of the signal as the defect size grows. These higher intensity impulses occur as the defect size increases and can sometimes excite structural natural frequencies, harmonics of other rotating parts, etc. which span a large frequency range and mask the original defective signal [8]. As a result, the Kurtosis value decreases, hindering the fault detection process. In

Table 3 it can be seen that the value of the maximum Kurtosis found by the Kurtogram decreases after D4, exactly at the same point when the SNR starts to decline.

The improvement in the crest factor for the smaller defects is especially interesting, due to the importance of detecting incipient faults during the early stages of degradation. In the original signals acquired for D2, D3 and D4 it is difficult to differentiate between peaks generated as a consequence of the bearing ball rolling over the defect from other signal components produced mainly by background noise. Nevertheless, in the signal processed using SK those peaks are much more visible over the background noise, which allows an easy detection and identification of the defects during the first stages of the degradation process. The bursts observed in D5 to D9 are already clearly visible in the original signals. As a consequence, the slight reduction in the CF for those cases (except D7) does not affect the detectability of the defect, which is still clearly visible in the filtered signals as well. In D7 the maximum Kurtosis found is curiously high compared with the values found in D5, D6, D8 and D9, and for that particular case the SNR was significantly improved in the filtered signal.

\section{Frequency domain}

In Figure 7 are represented the envelopes of the original (left) and filtered (right) signals for the 9 different defects tested. The same spikes remain after the filtration process and again it is evident that the background noise level has been reduced in the filtered signal envelopes. The CF represented in Figure 8 and detailed in Table 5 shows similar trends as showed in 
Figure 5 and Table 4, with a clear improvement of SNR in the smaller defects (D1 to D4) and D7, but worse results were obtained for the defects D5, D6, D8 and D9. No new peaks indicative of burst presence are visible in the envelope of the filtered signal for the defect D1 despite of an increment in the $\mathrm{CF}$ of $38.31 \%$. This result indicates that D1 is too small to be detected either in the time or the frequency domain even using SK.

Original

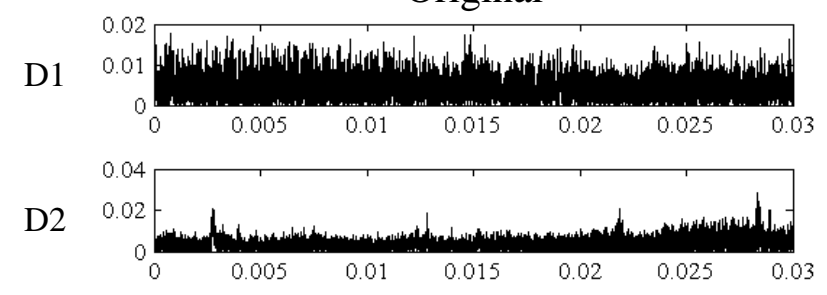

D3

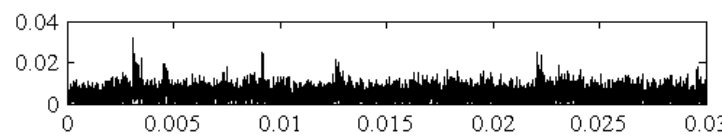

D4

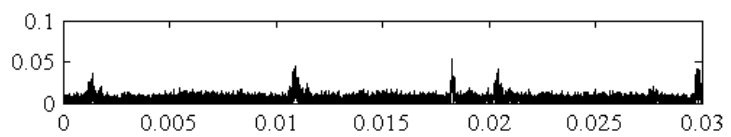

D5

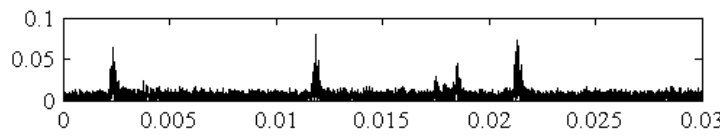

D6

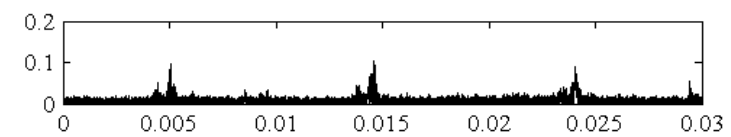

D7

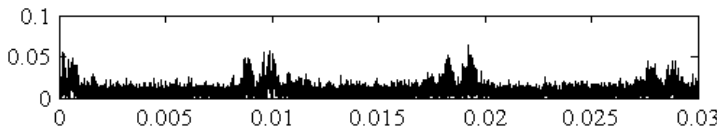

D8

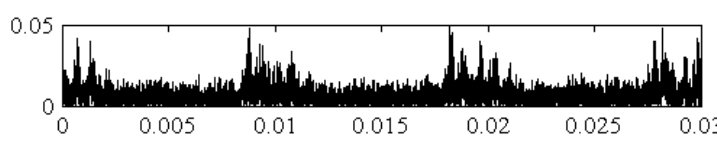

D9

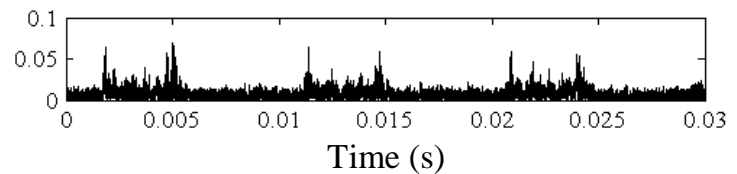

Filtered
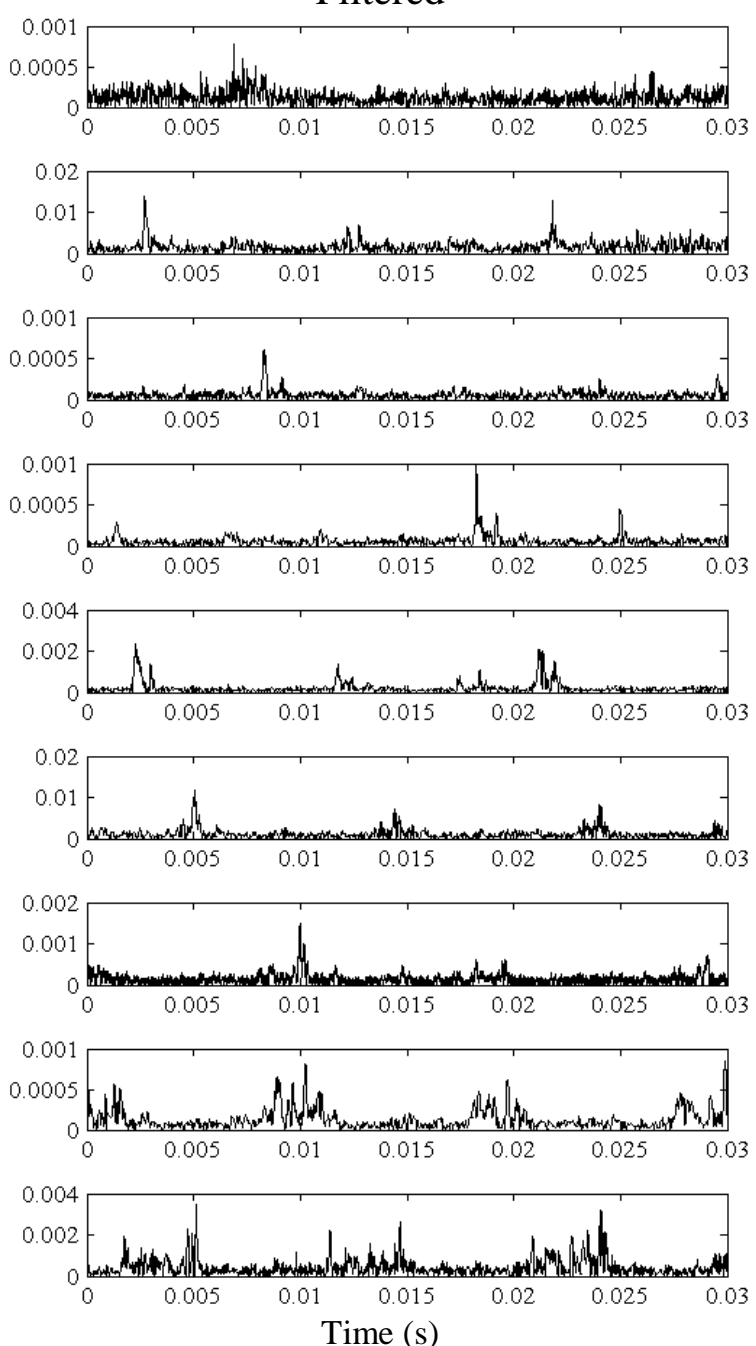

Figure 7.

Squared envelope of the original (left) and filtered signals (right) 
Table 5:

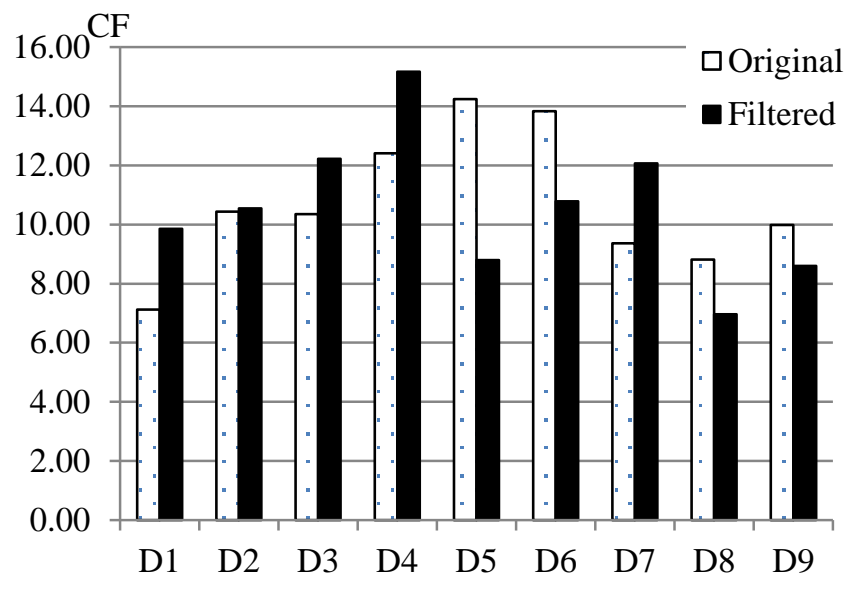

Figure 8.
$\mathrm{CF}$ for the original and filtered envelopes

\begin{tabular}{lccc} 
& Original & Filtered & Improvement \% \\
\hline D1 & 7.12 & 9.85 & 38.31 \\
D2 & 10.43 & 10.54 & 0.99 \\
D3 & 10.35 & 12.22 & 18.07 \\
D4 & 12.40 & 15.16 & 22.28 \\
D5 & 14.24 & 8.80 & -38.19 \\
D6 & 13.83 & 10.78 & -22.05 \\
D7 & 9.36 & 12.07 & 28.86 \\
D8 & 8.81 & 6.96 & -20.95 \\
D9 & 9.98 & 8.60 & -13.86 \\
\hline
\end{tabular}

Crest factor for the original and filtered envelopes

\section{Conclusion}

The denoising ability of SK when applied to AE signals has been tested in this work for an artificially damaged bearing. The capacity of SK to enhance the signature of a bearing fault is well known when applied to vibration signals, but only the work of Eftekharnejad, B. et al. [33] reported a successful application of SK to detect bearing faults using Acoustic Emission.

From the results obtained in this work it is possible to conclude that SK can be a very useful tool to reduce the background noise and improve the burst visibility in bearing $\mathrm{AE}$ signals obtained from defective bearings with different defect sizes. The improvement in the signal to noise ratio achieved was dependant on the defect size, obtaining a higher percentage of improvement in the smaller defects, precisely where the AE bursts where less visible in the original signal. This affirmation reinforces the recent findings of Eftekharnejad, B. et al. [33], confirming that SK can be helpful for the early identification of bearing defects using AE, especially during the early stages of degradation. The application of SK to AE signals can thus improve the detection rate of bearing faults and reduce the detection time once the degradation process starts. Nevertheless the results showed that it is not always possible to improve the signal to noise ratio for AE signals associated with an advanced stage of bearing damage.

\section{Acknowledgements}

Financial support from the Marie Curie FP7-ITN project "Energy savings from smart operation of electrical, process and mechanical equipment- ENERGY-SMARTOPS", Contract No: PITN-GA-2010-264940 is gratefully acknowledged.

\section{References}

[1] Chaturvedi, G. K. and Thomas, D. W. (1982), "Bearing fault detection using adaptive noise cancelling," TRANS.ASME J.MECH.DES., vol. 104, no. 2 , Apr. 1982, pp. 280-289. 
[2] Ho, D. and Randall, R. B. (2000), "Optimization of bearing diagnostic techniques using simulated and actual bearing fault signals," Mechanical Systems and Signal Processing, vol. 14, no. 5, pp. 763-788.

[3] Khemili, I. and Chouchane, M. (2005), "Detection of rolling element bearing defects by adaptive filtering," European Journal of Mechanics, A/Solids, vol. 24, no. 2, pp. 293-303.

[4] Patel, V. N., Tandon, N. and Pandey, R. K. (2012), "Improving defect detection of rolling element bearings in the presence of external vibrations using adaptive noise cancellation and multiscale morphology," Proceedings of the Institution of Mechanical Engineers, Part J: Journal of Engineering Tribology, vol. 226, no. 2, pp. 150-162.

[5] Moosavian, A., Ahmadi, H. and Tabatabaeefar, A. (2012), "Fault diagnosis of main engine journal bearing based on vibration analysis using Fisher linear discriminant, K-nearest neighbor and support vector machine," Journal of Vibroengineering, vol. 14, no. 2, pp. 894-906.

[6] Guo, Y., Liu, T. -., Na, J. and Fung, R. -. (2012), "Envelope order tracking for fault detection in rolling element bearings," Journal of Sound and Vibration.

[7] Antoni, J. (2006), "The spectral kurtosis: A useful tool for characterising non-stationary signals," Mechanical Systems and Signal Processing, vol. 20, no. 2, pp. 282-307.

[8] Antoni, J. and Randall, R. B. (2006), "The spectral kurtosis: Application to the vibratory surveillance and diagnostics of rotating machines," Mechanical Systems and Signal Processing, vol. 20, no. 2, pp. 308-331.

[9] Cong, F., Chen, J. and Dong, G. (2010), "Rolling bearing fault diagnosis based on spectral kurtosis in condition monitoring," COMADEM 2010 - Advances in Maintenance and Condition Diagnosis Technologies Towards Sustainable Society, Proc. 23rd Int. Congr. Condition Monitoring and Diagnostic Engineering Management, pp. 529.

[10] Komgom, C. N., Mureithi, N. W. and Lakis, A. A. (2008), "Application of time synchronous averaging, spectral kurtosis and support vector machines for bearing fault identification," American Society of Mechanical Engineers, Pressure Vessels and Piping Division (Publication) PVP, vol. 7, pp. 137.

[11] Shi, L., Randall, R. B. and Antoni, J. (2004), "Rolling element bearing fault detection using improved envelope analysis," IMechE Event Publications, vol. 2004 2, pp. 301.

[12] Al-Dossary, S., Hamzah, R. I. R. and Mba, D. (2009), "Observations of changes in acoustic emission waveform for varying seeded defect sizes in a rolling element bearing," Applied Acoustics, vol. 70, no. 1, pp. 58-81.

[13] Al-Ghamd, A. M. and Mba, D. (2006), "A comparative experimental study on the use of acoustic emission and vibration analysis for bearing defect identification and estimation of defect size," Mechanical Systems and Signal Processing, vol. 20, no. 7, pp. 1537-1571.

[14] Behzad, M., AlandiHallaj, A., Bastami, A. R., Eftekharnejad, B., Charnley, B. and Mba, D. (2009), "Defect size estimation in rolling element bearings using vibration time waveform," Insight: NonDestructive Testing and Condition Monitoring, vol. 51, no. 8, pp. 426-430.

[15] Elforjani, M. and Mba, D. (2010), "Accelerated natural fault diagnosis in slow speed bearings with Acoustic Emission," Engineering Fracture Mechanics, vol. 77, no. 1, pp. 112-127.

[16] Elforjani, M. and Mba, D. (2008), "Detecting the onset, propagation and location of non-artificial defects in a slow rotating thrust bearing with acoustic emission," Insight: Non-Destructive Testing and Condition Monitoring, vol. 50, no. 5, pp. 264-268.

[17] Hawman, M. W. and Galinaitis, W. S. (1988), "Acoustic emission monitoring of rolling element bearings," Ultrasonics Symposium Proceedings, Vol. 2, pp. 885. 
[18] Kilundu, B., Chiementin, X., Duez, J. and Mba, D. (2011), "Cyclostationarity of Acoustic Emissions (AE) for monitoring bearing defects," Mechanical Systems and Signal Processing, vol. 25, no. 6, pp. 20612072.

[19] Mba, D. and Rao, R. B. K. N. (2006), "Development of acoustic emission technology for condition monitoring and diagnosis of rotating machines: Bearings, pumps, gearboxes, engines, and rotating structures," Shock and Vibration Digest, vol. 38, no. 2, pp. 3-16.

[20] Shiroishi, J., Li, Y., Liang, S., Kurfess, T. and Danyluk, S. (1997), "Bearing condition diagnostics via vibration and acoustic emission measurements," Mechanical Systems and Signal Processing, vol. 11, no. 5, pp. 693-705.

[21] Yoshioka, T., Korenaga, A., Mano, H. and Yamamoto, T. (1999), "Diagnosis of rolling bearing by measuring time interval of AE generation," Journal of Tribology, vol. 121, no. 3, pp. 468-472.

[22] Al-Balushi, K. R., Addali, A., Charnley, B. and Mba, D. (2010), "Energy index technique for detection of acoustic emissions associated with incipient bearing failures," Applied Acoustics, vol. 71, no. 9, pp. 812-821.

[23] Chiementin, X., Mba, D., Charnley, B., Lignon, S. and Dron, J. P. (2010), "Effect of the denoising on acoustic emission signals," Journal of Vibration and Acoustics, Transactions of the ASME, vol. 132, no. 3 , pp. 0310091-0310099.

[24] Couturier, J. and Mba, D. (2008), "Operational bearing parameters and acoustic emission generation," Journal of Vibration and Acoustics, Transactions of the ASME, vol. 130, no. 2.

[25] Elforjani, M. and Mba, D. (2011), "Condition monitoring of slow-speed shafts and bearings with Acoustic emission," Strain, vol. 47, no. SUPPL. 2, pp. 350-363.

[26] Elforjani, M. and Mba, D. (2010), "Acoustic emissions observed from a naturally degrading slow speed bearing and shaft," COMADEM 2010 - Advances in Maintenance and Condition Diagnosis Technologies Towards Sustainable Society, Proc. 23rd Int. Congr. Condition Monitoring and Diagnostic Engineering Management, pp. 355.

[27] Elforjani, M. and Mba, D. (2009), "Assessment of natural crack initiation and its propagation in slow speed bearings," Nondestructive Testing and Evaluation, vol. 24, no. 3, pp. 261-275.

[28] Elforjani, M. and Mba, D. (2008), "Monitoring the onset and propagation of natural degradation process in a slow speed rolling element bearing with acoustic emission," Journal of Vibration and Acoustics, Transactions of the ASME, vol. 130, no. 4.

[29] Elforjani, M. and Mba, D. (2008), "Observations and location of acoustic emissions for a naturally degrading rolling element thrust bearing," Journal of Failure Analysis and Prevention, vol. 8, no. 4, pp. 370-385.

[30] Mba, D. (2008), "The use of acoustic emission for estimation of bearing defect size," Journal of Failure Analysis and Prevention, vol. 8, no. 2, pp. 188-192.

[31] Mirhadizadeh, S. A., Moncholi, E. P. and Mba, D. (2010), "Influence of operational variables in a hydrodynamic bearing on the generation of acoustic emission," Tribology International, vol. 43, no. 9, pp. 1760-1767.

[32] Tandon, N. and Nakra, B. C. (1992), "Comparison of vibration and acoustic measurement techniques for the condition monitoring of rolling element bearings," Tribology International, vol. 25, no. 3, pp. 205-212. 
[33] Eftekharnejad, B., Carrasco, M. R., Charnley, B. and Mba, D. (2011), "The application of spectral kurtosis on Acoustic Emission and vibrations from a defective bearing," Mechanical Systems and Signal Processing, vol. 25, no. 1, pp. 266-284.

[34] Matthews, J. R. (1983), Acoustic emission, New York : Gordon and Breach Science Publishers, c1983.

[35] Tan, C. K. and Mba, D. (2005), "Limitation of acoustic emission for identifying seeded defects in gearboxes," Journal of Nondestructive Evaluation, vol. 24, no. 1, pp. 11-28.

[36] Antoni, J. (2007), "Fast computation of the kurtogram for the detection of transient faults," Mechanical Systems and Signal Processing, vol. 21, no. 1, pp. 108-124.

[37] Antoni, J. , Jérôme Antoni personal page, available at: http://www.utc.fr/ antoni/ (accessed 24/07/2012).

[38] Williams, T., Ribadeneira, X., Billington, S. and Kurfess, T. (2001), "Rolling element bearing diagnostics in run-to-failure lifetime testing," Mechanical Systems and Signal Processing, vol. 15, no. 5, pp. 979-993. 